\title{
Modern Friedel-Crafts chemistry. Part 35. New synthetic approach to substituted indolo[2,1-a][2]benzazepines and indolo[2,1-a]isoquinolines via Friedel-Crafts cyclialkylations
}

\author{
Hassan A.K. Abd El-Aal,* Ali A. Khalaf, and Talaat I. El-Emary \\ Chemistry Department, Faculty of Science, Assiut University, Assiut, 71516, Egypt \\ E-mail: hassan272004@yahoo.com
}

\begin{abstract}
Facile procedures for the construction of fused indole-containing heteropolycycles 1a-f and 2a-c have been developed. The methodology involves Friedel-Crafts cyclialkylations of heteoraryl alkanols in the presence of both Brönsted (PPA) and Lewis $\left(\mathrm{AlCl}_{3} / \mathrm{CH}_{3} \mathrm{NO}_{2}\right)$ acid catalysts. The starting alkanols 6a-f and 12a-c were smoothly obtained both by reactions of the corresponding carboxylic acid esters and the corresponding ketones with Grignard reagents. Overall, this approach allows for easy and efficient access to polycyclic indoles from easily synthesized precursors.
\end{abstract}

Keywords: Friedel-Crafts cyclialkylation, heteropolycycles, 7-methyl-2-phenyl-1H-indole, heteroarylalkanols, Grignard reagents, 5,6-dihydro-8-methylindolo[2,1-a]isoquinoline

\section{Introduction}

Heteropolycycles containing indole moieties possess a wide diversity of biological activities ${ }^{1}$ beside their presence in a large array of pharmaceuticals ${ }^{2}$ and natural products. ${ }^{3}$ For example, 7,8-dihydro-6H-indolo[2,1-a][2] benzazepine (1) ${ }^{4}$ and 6,7-dihydroindolo[2,1-a]isoquinoline (2) ${ }^{5-7}$ have unique nitrogen-containing tetracyclic ${ }^{8}$ structures (Scheme 1). Their analogues occur widely in isolated natural products ${ }^{9}$, in $\operatorname{drugs}^{10}$ and the derived $\pi$-conjugated materials are used as organic semiconductors. ${ }^{11}$
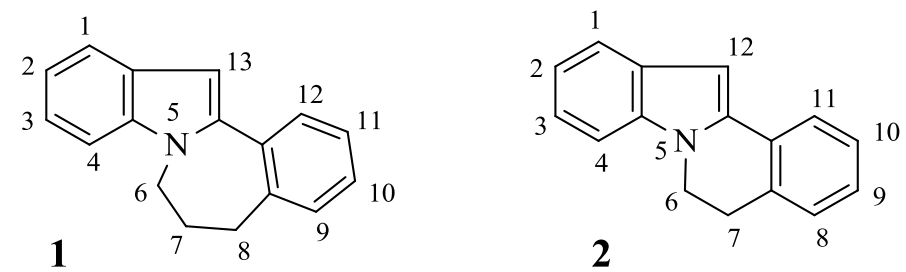

Scheme 1. Indole scaffolds containing heteropolycycles. 
The indole scaffold 1 was obtained early by Kozikowski et al. ${ }^{12,13}$ from the derived $N$ alkylindole and 2-bromobenzyl bromide in the presence of $\mathrm{Pd}\left(\mathrm{PPh}_{3}\right)_{4}$. Lee and co-workers ${ }^{14,15}$ reported the synthesis of benzazepinoindoles via intramolecular Heck type reaction by applying intramolecular palladium-catalyzed arylation of indole-containing Baylis-Hillman adducts. ${ }^{16}$ Orito et al. ${ }^{17}$ reported the formation of alkaloids of berberine and dibenzopyrrocoline series via the palladium-catalyzed coupling of amide derived from $o$-halobenzylisoquinolines. On the other hand, Sharma et al. ${ }^{18}$ reported interesting examples for the synthesis of benzazepinoindoles through the 7-endo trig Pictet-Spengler cyclization with various carbonyl compounds. ${ }^{19}$

Other strategies have been successfully applied in the synthesis of polyindoles. That included benzyne reactions ${ }^{20}$, oxidative couplings of 1-benzylisoquinoline ${ }^{21}$, enamine photocyclization ${ }^{22}$, silicon mediated ring closure of benzyl anion ${ }^{23}$, radical cyclization ${ }^{24}$ and palladium-catalyzed couplings. ${ }^{25}$

Faust et al. $^{5}$ failed to prepare the tetracyclic amine indoloisoquinoline (2) by the methodology of Kozikowski. However, they succeeded to obtain $\mathbf{2}$ via consecutive six-step reactions starting from 3-formylindoles in low overall yields. Other synthetic methodologies have been developed to generate these compounds by stepwise introduction or construction of the pyrrole and benzene rings. $^{26}$

The development of new direct, concise and economical routes to this class of compound is currently a popular research area for both medicinal and synthetic organic chemists. ${ }^{27,28}$ In this paper, we applied our experience in Friedel-Crafts cyclialkylations ${ }^{29-36}$ to offer unequivocal syntheses for both substituted 7,8-dihydro-6H-indolo[2,1-a][2]benzazepine (1) and 6,7-dihydroindolo[2,1-a]isoquinoline (2) via intramolecular ring closures of some synthesized heteroaryl alkanols.

\section{Results and Discussion}

\section{Synthesis of cyclialkylating alcohols}

The heteroaryl alkanols required for this work (6a-f and 12a-c) were all obtained starting from the easily accessible ${ }^{37}$ 7-methyl-2-phenyl-1H-indole (3) via three different pathways as formulated in Scheme 2: Path 1, included the base catalyzed N-alkylation of 3 with ethyl 3bromopropanoate (4) to give ethyl 3-(7-methyl-2-phenyl-1H-indol-1-yl)propanoate (5). This ester was allowed to react with two equivalents of Grignard reagents ${ }^{38-40}$ to afford the corresponding tertiary alcohols 6a-c $\left(\mathrm{R}^{1}=\mathrm{R}^{2}\right)$ (Table 1, Entries 1-3).

Path 2 comprised the conversion of substrate $\mathbf{3}$ to tertiary alcohols $\mathbf{6 d - f}$ via three consecutive steps: (i) cyanoethylation ${ }^{41}$ of $\mathbf{3}$ with acrylonitrile (7) in the presence of Triton B, (ii) reaction of the resulting nitrile $\mathbf{8}$ with one equivalent of Grignard reagents to afford ketones $\mathbf{9 a , b}$ and (iii) reaction of ketones $9 \mathbf{a}, \mathbf{b}$ with Grignard reagents to give alcohols $\mathbf{6} \mathbf{d}-\mathbf{f}\left(\mathrm{R}^{1} \# \mathrm{R}^{2}\right)$ (Table 1, Entries 4-6). 


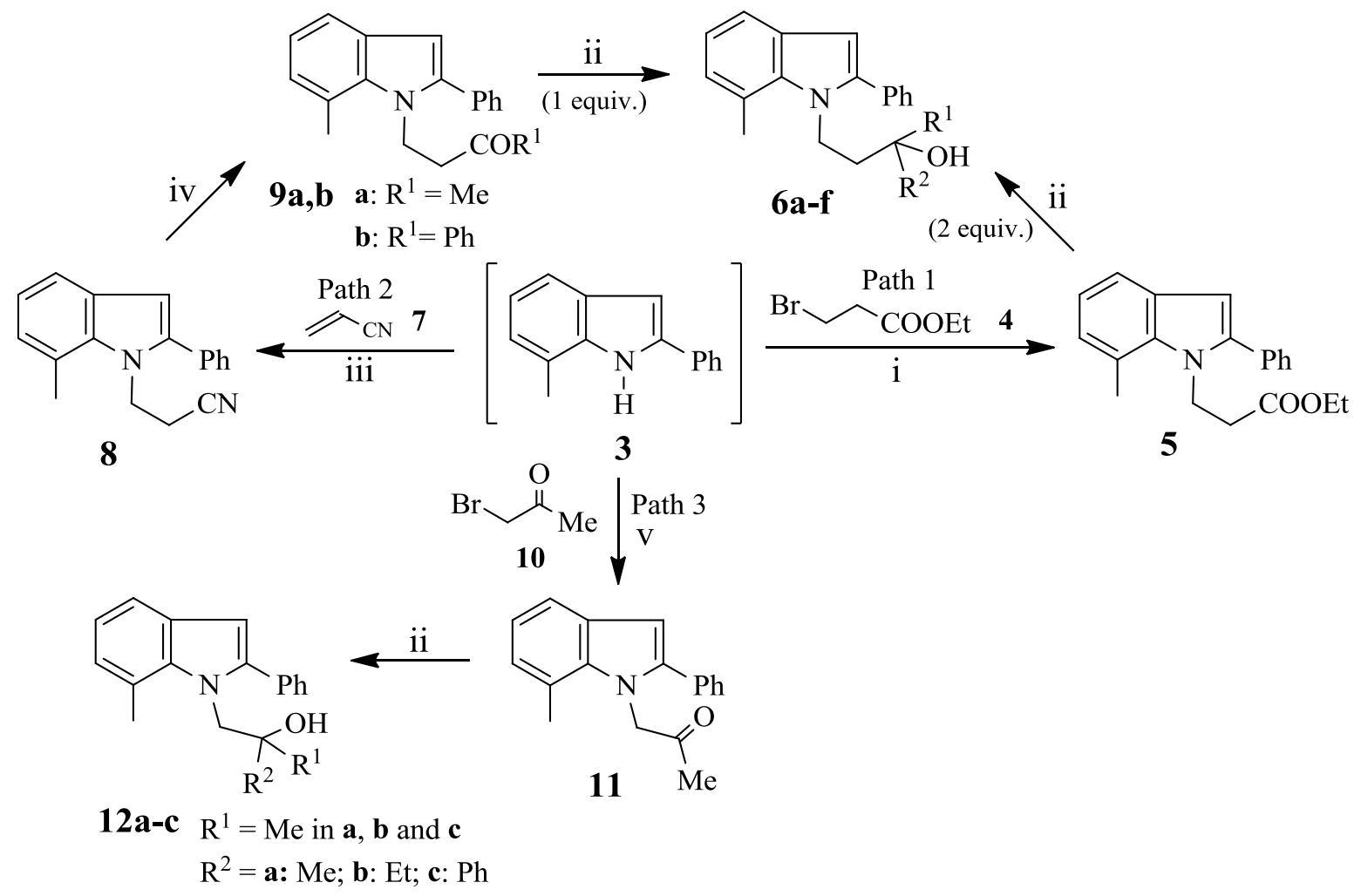

Scheme 2. Reagents and conditions: (i) $\mathrm{K}_{2} \mathrm{CO}_{3} / \mathrm{Cu}$, xylene, reflux $12 \mathrm{~h}, 85 \%$, (ii) $\mathrm{RMgX}, \mathrm{Et}_{2} \mathrm{O}$, $\mathrm{NH}_{4} \mathrm{Cl}$ soln, (Table 1), (iii) Triton $\mathrm{B}$, dioxane, $80-90^{\circ} \mathrm{C}, 5 \mathrm{~h}, 75 \%$, (iv) $\mathrm{RMgX}, \mathrm{Et}_{2} \mathrm{O} / \mathrm{PhH}, \mathrm{HCl}$, reflux, (v) $\mathrm{K}_{2} \mathrm{CO}_{3} / \mathrm{KI}$, acetone, $10 \mathrm{~h}, 78 \%$.

Table 1. Optimum conditions for the synthesis of heteroarylalkanols 6a-f and 12a-c

\begin{tabular}{|c|c|c|c|c|c|c|}
\hline Entry & Substrate & $\mathrm{R}^{1}$ & $\mathrm{R}^{2}$ & Conditions $^{\mathrm{a}}$ & $\mathrm{Mp}{ }^{\circ} \mathrm{C}\left(n_{D}^{25}\right)$ & Product $(\%)^{\mathrm{b}}$ \\
\hline 1 & 5 & $\mathrm{Me}$ & $\mathrm{Me}$ & $\mathrm{MeMgI}, \mathrm{Et}_{2} \mathrm{O}, \mathrm{rt}, 8 \mathrm{hr}$ & 110 & $\mathbf{6 a}(87)$ \\
\hline 2 & 5 & $\mathrm{Et}$ & Et & $\mathrm{EtMgBr}, \mathrm{Et}_{2} \mathrm{O}, \mathrm{rt}, 8 \mathrm{hr}$ & 83 & $6 \mathbf{b}(84)$ \\
\hline 3 & 5 & $\mathrm{Ph}$ & $\mathrm{Ph}$ & $\mathrm{PhMgBr}, \mathrm{Et}_{2} \mathrm{O}, \mathrm{rt}, 15 \mathrm{hr}$ & 142 & $\mathbf{6 c}(79)$ \\
\hline 4 & $9 a$ & $\mathrm{Me}$ & Et & $\mathrm{EtMgBr}, \mathrm{Et}_{2} \mathrm{O}, \mathrm{rt}, 9 \mathrm{hr}$ & 92 & $\mathbf{6 d}(91)$ \\
\hline 5 & $9 \mathrm{~b}$ & $\mathrm{Ph}$ & Et & $\mathrm{EtMgBr}, \mathrm{Et}_{2} \mathrm{O}, \mathrm{rt}, 13 \mathrm{hr}$ & 120 & $6 e(82)$ \\
\hline 6 & 9a & $\mathrm{Me}$ & $\mathrm{Ph}$ & $\mathrm{PhMgBr}, \mathrm{Et}_{2} \mathrm{O}, \mathrm{rt}, 10 \mathrm{hr}$ & 98 & 6f $(86)$ \\
\hline 7 & 11 & $\mathrm{Me}$ & $\mathrm{Me}$ & $\mathrm{MeMgI}, \mathrm{Et}_{2} \mathrm{O}, \mathrm{rt}, 8 \mathrm{hr}$ & 68 & 12a $(92)$ \\
\hline 8 & 11 & $\mathrm{Me}$ & Et & $\mathrm{EtMgBr}, \mathrm{Et}_{2} \mathrm{O}, \mathrm{rt}, 9 \mathrm{hr}$ & (1.568) & $\mathbf{1 2 b}(89)$ \\
\hline 9 & 11 & $\mathrm{Me}$ & $\mathrm{Ph}$ & $\mathrm{PhMgBr}, \mathrm{Et}_{2} \mathrm{O}, 15 \mathrm{hr}$ & $(1.584)$ & $\mathbf{1 2 c}(85)$ \\
\hline
\end{tabular}

${ }^{a}$ All reactions were performed using 0.2 equiv. excess of $\mathrm{RMgX}$. ${ }^{b}$ Isolated yield refer to substrate. 
The third route (path 3) encompassed the production of alcohols 12a-c. These were prepared smoothly through the N-alkylation of $\mathbf{3}$ with 1-bromopropan-2-one (10) to give 1-(7-methyl-2phenyl-1H-indol-1-yl)propan-2-one (11). The latter ketone was treated with Grignard reagents to afford alcohols 12a-c (Scheme 2, Table 1, Entries 7-9). The structures of all new alcohols were appropriately established by the usual spectroscopic methods.

Cyclialkylations producing substituted 7,8-dihydro-6H-indolo[2,1-a][2]benzazepines (1a-f). Cyclialkylations of alcohols 6a-f were conducted in the presence of $\mathrm{AlCl}_{3} / \mathrm{CH}_{3} \mathrm{NO}_{2}$ and PPA catalysts under the conditions outlined in Table 2 to give the title benzazepines 1a-f (Table 2 and Scheme 3).

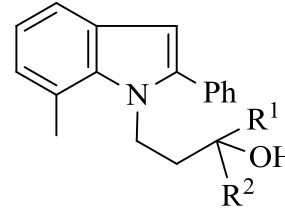

6a-f

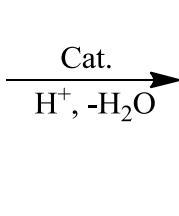
2

西

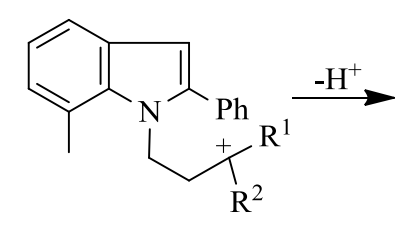

13a-f

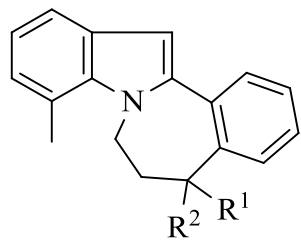

1a-f

Scheme 3. Cyclialkylations of heteroarylalkanols 6a-f.

Table 2. Cyclialkylation conditions and results of heteroarylalkanols 6a-f

\begin{tabular}{ccccccc}
\hline Entry & Substrate & Catalyst & Solvent & Temp. ${ }^{\circ} \mathrm{C}$ & Time (h) & Product (\%) ${ }^{\mathrm{a}}$ \\
\hline 1 & $\mathbf{6 a}$ & $\mathrm{AlCl}_{3} / \mathrm{CH}_{3} \mathrm{NO}_{2}{ }^{\mathrm{b}}$ & $\mathrm{DCM}^{\mathrm{c}}$ & $\mathrm{RT}$ & 2 & $\mathbf{1 a}(82)$ \\
2 & $\mathbf{6 a}$ & $\mathrm{PPA}^{\mathrm{d}}$ & -- & 160 & 2 & $\mathbf{1 a}(75)$ \\
3 & $\mathbf{6 b}$ & $\mathrm{AlCl}_{3} / \mathrm{CH}_{3} \mathrm{NO}_{2}$ & $\mathrm{DCM}$ & $\mathrm{RT}$ & 2 & $\mathbf{1 b}(81)$ \\
4 & $\mathbf{6 b}$ & $\mathrm{PPA}$ & -- & 160 & 1 & $\mathbf{1 b}(73)$ \\
5 & $\mathbf{6 c}$ & $\mathrm{AlCl}_{3} / \mathrm{CH}_{3} \mathrm{NO}_{2}$ & $\mathrm{DCM}$ & 50 & 52 & $\mathbf{1 c}(81)$ \\
6 & $\mathbf{6 c}$ & $\mathrm{PPA}$ & -- & 220 & 14 & $\mathbf{1 c}(76)$ \\
7 & $\mathbf{6 d}$ & $\mathrm{AlCl}_{3} / \mathrm{CH}_{3} \mathrm{NO}_{2}$ & $\mathrm{DCM}$ & $\mathrm{RT}$ & 2 & $\mathbf{1 d}(79)$ \\
8 & $\mathbf{6 d}$ & $\mathrm{PPA}$ & -- & 160 & 2 & $\mathbf{1 d}(74)$ \\
9 & $\mathbf{6 e}$ & $\mathrm{AlCl}_{3} / \mathrm{CH}_{3} \mathrm{NO}_{2}$ & $\mathrm{DCM}$ & $\mathrm{RT}$ & 2 & $\mathbf{1 e}(84)$ \\
10 & $\mathbf{6 e}$ & $\mathrm{PPA}_{10}$ & -- & 160 & 2 & $\mathbf{1 e}(74)$ \\
11 & $\mathbf{6 f}$ & $\mathrm{AlCl}_{3} / \mathrm{CH}_{3} \mathrm{NO}_{2}$ & $\mathrm{DCM}$ & $\mathrm{RT}$ & 4 & $\mathbf{1 f}(80)$ \\
12 & $\mathbf{6 f}$ & $\mathrm{PPA}^{2}$ & -- & 160 & 2 & $\mathbf{1 f}(76)$ \\
\hline
\end{tabular}

asolated yield refer to substrate. ${ }^{\mathrm{b}} \mathrm{With} \mathrm{AlCl}_{3} / \mathrm{CH}_{3} \mathrm{NO}_{2}$ catalyst reactant proportions were: carbinol (0.002 mole), $\mathrm{AlCl}_{3}(0.0024$ mole $), \mathrm{CH}_{3} \mathrm{NO}_{2}(0.024$ mole $)$, solvent $(10 \mathrm{ml})$. ${ }^{\mathrm{c}}$ Dichloromethane. ${ }^{\mathrm{d}}$ With PPA catalyst reactant proportions were: carbinol $(0.5 \mathrm{~g})$ and PPA $(5 \mathrm{~g})$. 
Cyclialkylation of alcohol $\mathbf{6 c}\left(\mathrm{R}^{1}, \mathrm{R}^{2}=\mathrm{Ph}\right)$ to 7,8-dihydro-4-methyl-8,8-diphenyl-6Hindolo[2,1-a][2]benzazepines (1c) was effected in the presence of acidic catalysts under more strenuous conditions. As evident from Table 2 (Entries 5 and 6), cyclialkylation of $\mathbf{6 c}$ with $\mathrm{AlCl}_{3} / \mathrm{CH}_{3} \mathrm{NO}_{2}$ required $52 \mathrm{~h}$ in DCM solution at $50^{\circ} \mathrm{C}$ and with PPA required $14 \mathrm{~h}$ at $220{ }^{\circ} \mathrm{C}$. That was attributed to the steric interactions exerted by both bulky phenyl groups at the closure step. $^{29-35}$

Cyclialkylations producing substituted 6,7-dihydroindolo[2,1-a]isoquinolines (2a-c). Cyclialkylations of alcohols 12a-c were smoothly carried out in the presence of $\mathrm{AlCl}_{3} / \mathrm{CH}_{3} \mathrm{NO}_{2}$ and PPA catalysts under different reaction conditions (Table 3, Scheme 4). Upon treatment of such alcohols with acidic catalysts, the generated stable tertiary carbocations 14a-c underwent closure to substituted 6,7-dihydroindolo[2,1-a]isoquinoline 2a-c in overall high reaction yields.

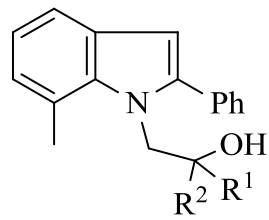

$12 \mathrm{a}-\mathbf{c}$
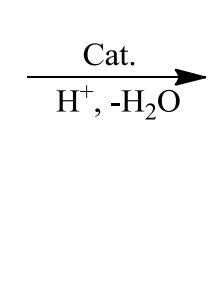

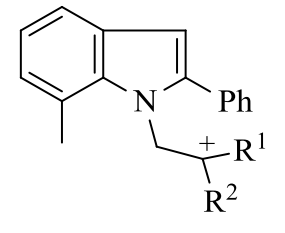

14a-c

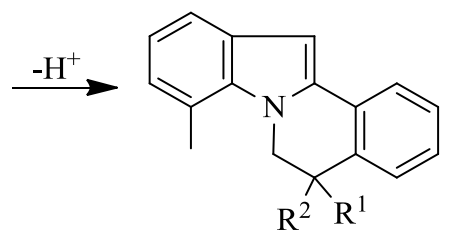

2a-c

Scheme 4. Cyclialkylations of heteroarylalkanols 12a-c.

Table 3. Cyclialkylation conditions and results of heteroarylalkanols 12a-c

\begin{tabular}{ccccccc}
\hline Entry & Substrate & Catalyst & Solvent & Temp. ${ }^{\circ} \mathrm{C}$ & Time (h) & Product (\%) \\
\hline 1 & $\mathbf{1 2 a}$ & $\mathrm{AlCl}_{3} / \mathrm{CH}_{3} \mathrm{NO}_{2}$ & $\mathrm{DCM}$ & $\mathrm{RT}$ & 3 & $\mathbf{2 a}(82)$ \\
2 & $\mathbf{1 2 a}$ & $\mathrm{PPA}$ & -- & 140 & 1 & $\mathbf{2 a}(76)$ \\
3 & $\mathbf{1 2 b}$ & $\mathrm{AlCl}_{3} / \mathrm{CH}_{3} \mathrm{NO}_{2}$ & $\mathrm{DCM}$ & $\mathrm{RT}$ & 3 & $\mathbf{2 b}(85)$ \\
4 & $\mathbf{1 2 b}$ & $\mathrm{PPA}$ & -- & 140 & 1.5 & $\mathbf{2 b}(73)$ \\
5 & $\mathbf{1 2 c}$ & $\mathrm{AlCl}_{3} / \mathrm{CH}_{3} \mathrm{NO}_{2}$ & $\mathrm{DCM}$ & $\mathrm{RT}$ & 5 & $\mathbf{2 c}(79)$ \\
6 & $\mathbf{1 2 c}$ & $\mathrm{PPA}$ & -- & 160 & 1 & $\mathbf{2 c}(78)$ \\
\hline
\end{tabular}

Before closing this discussion, it is worthwhile to contrast the results of this work in which the cyclialkylating substrates (6a-f and 12a-c) include a 7-methyl-2-phenyl-1H-indol-1-yl moiety with a previous case ${ }^{36}$ in which the 7-methyl was missing. In the present work, electrophilic ring closure occurred exclusively at the 2-phenyl group yielding either benzazepine or isoquinoline derivatives. In the previous case, however, closure occurred at the 7-position (which is not blocked) to yield the respective quinoline derivative (Scheme 5). That proofs, however, that attack on the 7-position of the indolyl moiety, if not blocked, is favored over attack on the phenyl group that is attached to the 2-position of the indolyl moiety. Moreover, the fact that the ${ }^{1} \mathrm{H}$ 
NMR chemical shift of the methyl group remained nearly constant suggests that ipso-substitution is not involved.

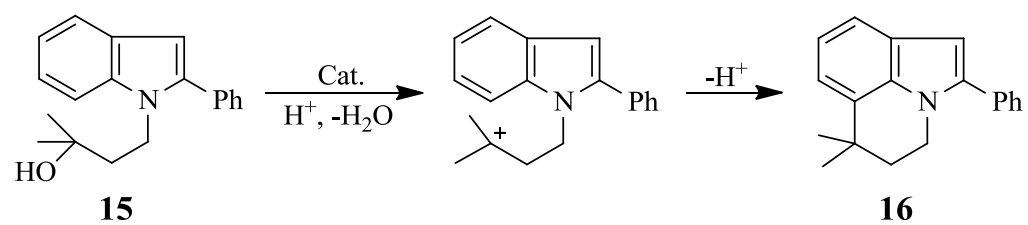

Scheme 5. Literature conversion of 2-methyl-4-(2-phenyl-1H-indol-1-yl)butan-2-ol (15) to 6,6-dimethyl-2-phenyl-5,6-dihydro-4H-pyrrolo[3,2,1-ij]quinoline (16).

\section{Conclusions}

We have developed new, simple and facile synthetic pathways for the construction of different indole-based substituted heteropolycycles via Friedel-Crafts cyclialkylation of heteroarylalkanols (6a-f and 12a-c) catalyzed by $\mathrm{AlCl}_{3} / \mathrm{CH}_{3} \mathrm{NO}_{2}$ and PPA. To the best of our knowledge, this is the first time that such novel substituted tetracycles (1a-f and 2a-c) have been described. The results show Friedel-Crafts cyclialkylations to be useful pathways to the syntheses of heteropolycycles.

\section{Experimental Section}

General. Unless otherwise indicated all common reagents and solvents were used as obtained from commercial suppliers without further purification. Melting points were measured on a digital Gallenkamp capillary melting point apparatus. The IR spectra were determined with a Shimadzu 470 Infrared spectrophotometer using $\mathrm{KBr}$ wafer and thin film techniques $\left(v \mathrm{~cm}^{-1}\right)$. The ${ }^{1} \mathrm{H}$ NMR spectra were recorded by JEOL LA $400 \mathrm{MHz}$ FT-NMR (400 MHz) and Varian 90 $\mathrm{MHz}$ NMR spectrometer using $\mathrm{CDCl}_{3}$ solvent with TMS as internal standard. Chemical shifts $(\delta)$ and $J$ values are reported in ppm and $\mathrm{Hz}$, respectively. Elemental analyses were performed on a Perkin-Elmer 2400 Series II analyzer. The mass spectra were performed by JEOL JMS 600 spectrometer at an ionizing potential of $70 \mathrm{eV}$ using the direct inlet system. The refractive index was measured using a Schmidt Haensch apparatus. Reactions were monitored by thin layer chromatography (TLC) using precoated silica plates visualized with UV light. Flash column chromatography was performed on basic alumina (E. Merck) with suitable eluents.

Ethyl 3-(7-methyl-2-phenyl-1H-indol-1-yl)propanoate (5). A mixture of 7-methyl-2-phenyl$1 H$-indole 3 (4.1 g, $20 \mathrm{mmol})$, ethyl 3-bromopropanoate $4(5 \mathrm{~g}, 3.4 \mathrm{~mL}, 28 \mathrm{mmol})$, anhydrous $\mathrm{K}_{2} \mathrm{CO}_{3}(1.6 \mathrm{~g}, 12 \mathrm{mmol})$ and a catalytic amount of $\mathrm{Cu}$-metal $(0.3 \mathrm{~g})$ in xylene $(25 \mathrm{~mL})$ was refluxed for $12 \mathrm{~h}$. Afterwards the solvent was removed by steam distillation and the residue was 
diluted with water and extracted with ether $(3 \times 40 \mathrm{~mL})$. The combined ethereal extracts were washed with water, dried over $\mathrm{MgSO}_{4}$, filtered, and concentrated to give crude oily ester $(5.2 \mathrm{~g}$, $85.5 \%$ ). Purification by flash column chromatography (basic alumina, EtOAc/n-hexane, 1/1) gave pure ethyl 3-(7-methyl-2-phenyl-1H-indol-1-yl)propanoate (5) $(4.9 \mathrm{~g}, 80.6 \%)$ as a pale yellow oil; $n_{D}^{25}$ 1.571. IR (film) $v_{\max } 3050,2985,1745,1600,1560,1490,1455,1320,1170$, $745 \mathrm{~cm}^{-1} ;{ }^{1} \mathrm{H} \mathrm{NMR}\left(90 \mathrm{MHz}, \mathrm{CDCl}_{3}, \mathrm{ppm}\right), \delta 1.30\left(3 \mathrm{H}, \mathrm{t}, J 9.0 \mathrm{~Hz}, \mathrm{CH}_{3}\right), 2.20\left(3 \mathrm{H}, \mathrm{s}, \mathrm{CH}_{3}\right)$, $2.70\left(2 \mathrm{H}, \mathrm{s}, J 9.0 \mathrm{~Hz}, \mathrm{CH}_{2}\right), 4.10\left(2 \mathrm{H}, \mathrm{t}, J 7.5 \mathrm{~Hz}, \mathrm{CH}_{2}\right), 4.30\left(2 \mathrm{H}, \mathrm{t}, J 7.5 \mathrm{~Hz}, \mathrm{CH}_{2}\right), 6.50(1 \mathrm{H}$, $\mathrm{s}, \mathrm{CH})$ and 6.90-7.70 ppm (8H, m, Ar-H); Anal. Calcd. for $\mathrm{C}_{20} \mathrm{H}_{21} \mathrm{NO}_{2}$ (307); C, 78.17; H, 6.84; N, 4.56. Found; C, 78.20; H, 6.88; N, 4.23\%.

3-(7-Methyl-2-phenyl-1H-indol-1-yl)propanenitrile (8). To an ice-cold solution of 7-methyl-2phenyl-1H-indole $3(5.1 \mathrm{~g}, 25 \mathrm{mmol})$ and acrylonitrile $7(4 \mathrm{~g}, 5 \mathrm{~mL}, 75 \mathrm{mmol})$ in dioxane (20 $\mathrm{mL}$ ) was treated with $0.5 \mathrm{ml}$ of Triton B. The reaction mixture was heated in a steam bath at 80$90{ }^{\circ} \mathrm{C}$ for $5 \mathrm{~h}$ and then it was concentrated. The residue was triturated with methanol $(3 \times 5 \mathrm{~mL})$ and the solid product was filtered off, washed copiously with water, and dried to give crude nitrile (4.8 g, 75\%). Crystallization from acetone gave pure nitrile 8 (4.3 g, 67\%) as white crystals, mp $102{ }^{\circ} \mathrm{C}$. IR (KBr): $v_{\max } 3070,2985,2250,1580,1475,1450,1320,1175,740 \mathrm{~cm}^{-1}$; ${ }^{1} \mathrm{H}$ NMR (90 MHz, $\left.\mathrm{CDCl}_{3}, \mathrm{ppm}\right), \delta 2.30\left(3 \mathrm{H}, \mathrm{s}, \mathrm{CH}_{3}\right), 2.80\left(2 \mathrm{H}, \mathrm{t}, J 7.5 \mathrm{~Hz}, \mathrm{CH}_{2}\right), 4.20(2 \mathrm{H}, \mathrm{t}, J$ $\left.7.5 \mathrm{~Hz}, \mathrm{CH}_{2}\right), 6.50(1 \mathrm{H}, \mathrm{s}, \mathrm{CH})$ and 6.90-7.70 (8H, m, Ar-H);Anal. Calcd. for $\mathrm{C}_{18} \mathrm{H}_{16} \mathrm{~N}_{2}(260)$; C, 83.07; H, 6.15; N, 10.76. Found; C, 82.75; H, 6.2; N, $10.93 \%$.

\section{Synthesis of heteroaryl ketones 9a,b. General procedure}

To an ice-cold Grignard reagent obtained as usual ${ }^{40}$ from $\mathrm{Mg}$ turnings ( $\left.0.24 \mathrm{~g}, 10 \mathrm{mmol}\right)$, alkyl or aryl halide $(10 \mathrm{mmol})$ in ether $(30 \mathrm{~mL})$ was added with stirring a solution of nitrile 8 (2 $\mathrm{g}, 8$ $\mathrm{mmol})$ in benzene $(30 \mathrm{~mL})$ over $10 \mathrm{~min}$. After complete addition, the solution was refluxed for $10 \mathrm{hr}$ then it was poured with srirring into ice-cold hydrochloric acid $(100 \mathrm{~mL}, 30 \%)$. The organic solvent was evaporated and the mixture was concentrated. The resulted mixture was then hydrolyzed by refluxing with a mixture of (benzene, $20 \mathrm{~mL}$ and $\mathrm{HCl}, 10 \mathrm{~mL}$ ) for $10 \mathrm{~h}$. The solution was cold and benzene layer was separated, while the aqueous layer was basified by vertually addition of solid $\mathrm{Na}_{2} \mathrm{CO}_{3}$ with stirring and then extracted with benzene $(2 \times 30 \mathrm{~mL})$. The combined benzene extracts were washed with water, dried over anhydrous $\mathrm{Na}_{2} \mathrm{SO}_{4}$ and the solvent was removed to afford the crude ketones $\mathbf{9 a , b}$ which were purified by crystallization. The spectral data of the ketones $\mathbf{9 a , b}$ are given below.

4-(7-Methyl-2-phenyl-1H-indol-1-yl)butan-2-one (9a). Yield 82\%; mp 73 $\mathrm{C}$ (ethanol); IR $(\mathrm{KBr}) v_{\max } 3060,2975,1745,1580,1485,1450,1365,1285,1070,1030,750,695 \mathrm{~cm}^{-1} ;{ }^{1} \mathrm{H}$ NMR (90 MHz, $\left.\mathrm{CDCl}_{3}, \mathrm{ppm}\right), \delta 2.10\left(3 \mathrm{H}, \mathrm{s}, \mathrm{CH}_{3}\right), 2.30\left(3 \mathrm{H}, \mathrm{s}, \mathrm{CH}_{3}\right), 2.80(2 \mathrm{H}, \mathrm{t}, J \mathrm{~J} .5 \mathrm{~Hz}$, $\left.\mathrm{CH}_{2}\right), 4.10\left(2 \mathrm{H}, \mathrm{t}, J\right.$ 7.5 Hz, $\left.\mathrm{CH}_{2}\right), 6.50(1 \mathrm{H}, \mathrm{s}, \mathrm{CH})$ and 6.80-7.60 (8H, m, Ar-H); Anal. Calcd. for $\mathrm{C}_{19} \mathrm{H}_{19} \mathrm{NO}$ (277); C, 82.31; H, 6.85; N, 5.05. Found; C, 81.90; H, 6.70; N, 5.15\%.

3-(7-Methyl-2-phenyl-1H-indol-1-yl)-1-phenylpropan-1-one (9b): Yield 88\%; mp $102{ }^{\circ} \mathrm{C}$ (methanol). IR (KBr) $v_{\max } 3070,2980,1705,1570,1475,1455,1370,1060,1030,735 \mathrm{~cm}^{-1} ;{ }^{1} \mathrm{H}$ NMR (90 MHz, $\left.\mathrm{CDCl}_{3}, \mathrm{ppm}\right), \delta 2.20\left(3 \mathrm{H}, \mathrm{s}, \mathrm{CH}_{3}\right), 2.90\left(2 \mathrm{H}, \mathrm{t}, J\right.$ 7.5 Hz, $\left.\mathrm{CH}_{2}\right), 4.0(2 \mathrm{H}, \mathrm{t}, J 7.5$ 
$\left.\mathrm{Hz}, \mathrm{CH}_{2}\right), 6.50(1 \mathrm{H}, \mathrm{s}, \mathrm{CH})$ and 6.80-7.50 (13H, m, Ar-H); Anal. Calcd. for $\mathrm{C}_{24} \mathrm{H}_{21} \mathrm{NO}$ (339); C, 84.95; H, 6.19; N, 4.12. Found; C, 84.77; H, 6.30; N, 4.43\%.

\section{Synthesis of 1-(7-methyl-2-phenyl-1H-indol-1-yl)propan-2-one (11)}

To a stirred mixture of compound 3 (4.1 g, $20 \mathrm{mmol})$, anhydrous $\mathrm{K}_{2} \mathrm{CO}_{3}(1.6 \mathrm{~g}, 12 \mathrm{mmol})$ and a catalytic amount of potassium iodide $(0.2 \mathrm{~g})$ in dry acetone $(30 \mathrm{~mL})$ was added dropwise a solution of bromoacetone $\mathbf{1 0}(3.8 \mathrm{~g}, 28 \mathrm{mmol})$ in dry acetone $(20 \mathrm{~mL})$ at reflux temperature. Reflux was continued for $10 \mathrm{~h}$. The reaction mixture was concentrated to dryness and then transferred to ice water $(100 \mathrm{~mL})$. The separated solid was collected by filtration and crystallized from acetone to yield ketone $11(4 \mathrm{~g}, 78 \%)$ as faint yellow crystals, $\mathrm{m} \mathrm{p} 118^{\circ} \mathrm{C}$. IR $(\mathrm{KBr}) v_{\max }$ 3070, 3015, 2985, 1745, 1580, 1470, 1440, 1370, 1060, 740, $690 \mathrm{~cm}^{-1}$; ${ }^{1} \mathrm{H}$ NMR (90 MHz, $\left.\mathrm{CDCl}_{3}, \mathrm{ppm}\right), \delta 2.10\left(3 \mathrm{H}, \mathrm{s}, \mathrm{CH}_{3}\right), 2.40\left(3 \mathrm{H}, \mathrm{s}, \mathrm{CH}_{3}\right), 4.90\left(2 \mathrm{H}, \mathrm{s}, \mathrm{CH}_{2}\right), 6.60(1 \mathrm{H}, \mathrm{s}, \mathrm{CH})$ and 6.90-7.90 (8H, m, Ar-H); Anal. Calcd. for $\mathrm{C}_{18} \mathrm{H}_{17} \mathrm{NO}$ (263); C, 82.12; H, 6.46; N, 5.32. Found; C, $81.85 ; \mathrm{H}, 6.42 ; \mathrm{N}, 5.40 \%$.

\section{Synthesis of alcohols 6a-f and 12a-c. General procedure}

To an ice-cold Grignard reagent solution obtained as usual ${ }^{38,39}$ from $\mathrm{Mg}$ turnings $(0.2 \mathrm{~g}, 8 \mathrm{mmol})$, alkyl-or aryl halide $(8 \mathrm{mmol})$ in ether $(25 \mathrm{~mL})$, was added a solution of ester 5 ( $1 \mathrm{~g}, 3.3 \mathrm{mmol})$ and/or ketones $\mathbf{9 a , b}$ or $\mathbf{1 1}(6.6 \mathrm{mmol})$ in ether $(30 \mathrm{~mL})$. the reaction mixture was stirred at required temperature for appointed time (Table 1) followed by decomposition with sat. aq. $\mathrm{NH}_{4} \mathrm{Cl}$ soln. The product was extracted with ether $(3 \times 30 \mathrm{~mL})$ and the combined organic phases were washed with water, dried over anhydrous $\mathrm{Na}_{2} \mathrm{SO}_{4}$ and the solvent was evaporated. The residue was purified by flash column chromatography (basic alumina, EtOAc/n-hexane, 1/1) gave the pure product 6a-f and 12a-c. The conditions and yields are shown in Table 1 and spectral data are given below:

2-Methyl-4-(7-methyl-2-phenyl-1H-indol-1-yl)butan-2-ol (6a). White crystals, mp $110{ }^{\circ} \mathrm{C}$ (methanol). IR (KBr) $v_{\max } 3385,3080,2960,2850,1580,1470,1450,1440,1330,915,750,670$ $\mathrm{cm}^{-1} ;{ }^{1} \mathrm{H} \mathrm{NMR}\left(90 \mathrm{MHz}, \mathrm{CDCl}_{3}, \mathrm{ppm}\right), \delta 1.20\left(6 \mathrm{H}, \mathrm{s}, 2 \mathrm{CH}_{3}\right), 1.90\left(2 \mathrm{H}, \mathrm{t}, J 7.5 \mathrm{~Hz}, \mathrm{CH}_{2}\right), 2.20$ $\left(3 \mathrm{H}, \mathrm{s}, \mathrm{CH}_{3}\right), 3.10\left(1 \mathrm{H}, \mathrm{s}, \mathrm{OH}\right.$ exchangeable with $\left.\mathrm{D}_{2} \mathrm{O}\right), 3.70\left(2 \mathrm{H}, \mathrm{t}, J 7.5 \mathrm{~Hz}, \mathrm{CH}_{2}\right), 6.60(1 \mathrm{H}, \mathrm{s}$, $\mathrm{CH}$ ) and 7.0-8.0 (8H, m, Ar-H); Anal. Calcd. for $\mathrm{C}_{20} \mathrm{H}_{23} \mathrm{NO}$ (293); C, 81.91; H, 7.85; N, 4.77. Found; C, 81.57; H, 8.20; N, 4.95\%.

3-Ethyl-1-(7-methyl-2-phenyl-1H-indol-1-yl)pentan-3-ol (6b). White plates, mp $83{ }^{\circ} \mathrm{C}$ (ethanol). IR (KBr) $v_{\max } 3420,3070,2950,1600,1580,1470,1450,1330,750,675 \mathrm{~cm}^{-1} ;{ }^{1} \mathrm{H}$ NMR (90 MHz, $\left.\mathrm{CDCl}_{3}, \mathrm{ppm}\right), \delta 0.90\left(6 \mathrm{H}, \mathrm{t}, J 9.0 \mathrm{~Hz}, 2 \mathrm{CH}_{3}\right), 1.40\left(4 \mathrm{H}, \mathrm{m}, J 9.0 \mathrm{~Hz}, 2 \mathrm{CH}_{2}\right)$, $1.90\left(2 \mathrm{H}, \mathrm{t}, J 7.5 \mathrm{~Hz}, \mathrm{CH}_{2}\right), 2.10\left(1 \mathrm{H}, \mathrm{s}, \mathrm{OH}\right.$ exchangeable with $\left.\mathrm{D}_{2} \mathrm{O}\right), 2.20\left(3 \mathrm{H}, \mathrm{s}, \mathrm{CH}_{3}\right), 3.70$ $\left(2 \mathrm{H}, \mathrm{t}, J 7.5 \mathrm{~Hz}, \mathrm{CH}_{2}\right), 6.60(1 \mathrm{H}, \mathrm{s}, \mathrm{CH})$ and 6.80-7.90 $(8 \mathrm{H}, \mathrm{m}, \mathrm{Ar}-\mathrm{H})$; Anal. Calcd. for $\mathrm{C}_{22} \mathrm{H}_{27} \mathrm{NO}(321) ; \mathrm{C}, 82.24 ; \mathrm{H}, 8.41 ; \mathrm{N}, 4.36$. Found; C, 82.50; H, 8.37; N, 4.70\%.

3-(7-Methyl-2-phenyl-1H-indol-1-yl)-1,1-diphenylpropan-1-ol (6c). White crystals, mp 142 ${ }^{\circ} \mathrm{C}$ (methanol). IR (KBr) $v_{\max } 3370,3090,2910,1610,1585,1475,1440,1330,755,670 \mathrm{~cm}^{-1}$; ${ }^{1} \mathrm{H}$ NMR $\left(90 \mathrm{MHz}, \mathrm{CDCl}_{3}, \mathrm{ppm}\right), \delta 2.30\left(3 \mathrm{H}, \mathrm{s}, \mathrm{CH}_{3}\right), 2.50\left(1 \mathrm{H}, \mathrm{s}, \mathrm{OH}\right.$ exchangeable with $\left.\mathrm{D}_{2} \mathrm{O}\right)$, 
$2.60(2 \mathrm{H}, \mathrm{t}, J$ 7.5 Hz, CH 2$), 3.90(2 \mathrm{H}, \mathrm{t}, J$ 7.5 Hz, CH$), 6.70(1 \mathrm{H}, \mathrm{s}, \mathrm{CH})$ and 7.10-7.90 (18H, m, Ar-H); Anal. Calcd. for $\mathrm{C}_{30} \mathrm{H}_{27} \mathrm{NO}$ (417); C, 86.33; H, 6.47; N, 3.35. Found; C, 86.37; H, $6.62 ; \mathrm{N}, 3.25 \%$.

3-Methyl-1-(7-methyl-2-phenyl-1H-indol-1-yl)pentan-3-ol (6d). White crystals, mp $92{ }^{\circ} \mathrm{C}$ (ethanol). IR (KBr) $v_{\max } 3385,3090,2990,1600,1590,1480,1460,1440,1330,760,675 \mathrm{~cm}^{-1}$; ${ }^{1} \mathrm{H}$ NMR (90 MHz, $\left.\mathrm{CDCl}_{3}, \mathrm{ppm}\right), \delta 0.80\left(3 \mathrm{H}, \mathrm{t}, J 9.0 \mathrm{~Hz}, \mathrm{CH}_{3}\right), 1.30\left(3 \mathrm{H}, \mathrm{s}, \mathrm{CH}_{2}\right), 1.40(2 \mathrm{H}, \mathrm{q}, J$ $\left.9.0 \mathrm{~Hz}, \mathrm{CH}_{2}\right), 1.70\left(1 \mathrm{H}, \mathrm{s}, \mathrm{OH}\right.$ exchangeable with $\left.\mathrm{D}_{2} \mathrm{O}\right), 2.20\left(3 \mathrm{H}, \mathrm{s}, \mathrm{CH}_{3}\right), 1.80(2 \mathrm{H}, \mathrm{t}, J \mathrm{~J} .5 \mathrm{~Hz}$, $\left.\mathrm{CH}_{2}\right), 3.70\left(2 \mathrm{H}, \mathrm{t}, J\right.$ 7.5 Hz, $\left.\mathrm{CH}_{2}\right), 6.60(1 \mathrm{H}, \mathrm{s}, \mathrm{CH})$ and 6.80-7.90 (8H, m, Ar-H); Anal. Calcd. for $\mathrm{C}_{21} \mathrm{H}_{25} \mathrm{NO}$ (307); C, 82.08; H, 8.14; N, 4.56. Found; C, 82.42; H, 8.09; N, 4.28\%.

1-(7-Methyl-2-phenyl-1H-indol-1-yl)-3-phenylpentan-3-ol (6e). Pale yellow needles, mp 120 ${ }^{\circ} \mathrm{C}\left(1: 3\right.$ benzene/ PE $\left.60-80{ }^{\circ} \mathrm{C}\right) . \mathrm{IR}(\mathrm{KBr}) v_{\max } 3340,3080,2985,1615,1570,1470,1460,1445$, 1330, $760 \mathrm{~cm}^{-1} ;{ }^{1} \mathrm{H} \mathrm{NMR}\left(90 \mathrm{MHz}, \mathrm{CDCl}_{3}, \mathrm{ppm}\right), \delta 0.90\left(3 \mathrm{H}, \mathrm{t}, J 9.0 \mathrm{~Hz}, \mathrm{CH}_{3}\right), 1.70(2 \mathrm{H}, \mathrm{q}, J$ $\left.9.0 \mathrm{~Hz}, \mathrm{CH}_{3}\right), 2.10\left(2 \mathrm{H}, \mathrm{t}, J 7.5 \mathrm{~Hz}, \mathrm{CH}_{2}\right), 2.20\left(1 \mathrm{H}, \mathrm{s}, \mathrm{OH}\right.$ exchangeable with $\left.\mathrm{D}_{2} \mathrm{O}\right), 2.30(3 \mathrm{H}, \mathrm{s}$, $\left.\mathrm{CH}_{3}\right), 3.80\left(2 \mathrm{H}, \mathrm{t}, J\right.$ 7.5 Hz, $\left.\mathrm{CH}_{2}\right), 6.60(1 \mathrm{H}, \mathrm{s}, \mathrm{CH})$ and 6.80-7.90 (13H, m, Ar-H); Anal. Calcd. for $\mathrm{C}_{26} \mathrm{H}_{27} \mathrm{NO}$ (369); C, 84.55; H, 7.04; N, 3.94. Found; C, 84.82; H, 7.36; N, 4.27\%.

4-(7-Methyl-2-phenyl-1H-indol-1-yl)-2-phenylbutan-2-ol (6f). White plates, mp $98{ }^{\circ} \mathrm{C}$ (ethanol). IR (KBr) $v_{\max } 3360,3090,2970,1600,1580,1450,1440,1330,745 \mathrm{~cm}^{-1} ;{ }^{1} \mathrm{H}$ NMR (90 MHz, $\left.\mathrm{CDCl}_{3}, \mathrm{ppm}\right), \delta 1.50\left(3 \mathrm{H}, \mathrm{s}, \mathrm{CH}_{3}\right), 2.20\left(2 \mathrm{H}, \mathrm{t}, J 7.5 \mathrm{~Hz}, \mathrm{CH}_{2}\right), 2.30\left(3 \mathrm{H}, \mathrm{s}, \mathrm{CH}_{3}\right), 2.50$ $\left(1 \mathrm{H}, \mathrm{s}, \mathrm{OH}\right.$ exchangeable with $\left.\mathrm{D}_{2} \mathrm{O}\right), 3.80\left(2 \mathrm{H}, \mathrm{t}, J\right.$ 7.5 Hz, $\left.\mathrm{CH}_{2}\right), 6.70(1 \mathrm{H}, \mathrm{s}, \mathrm{CH})$ and 6.80-8.0 (13H, m, Ar-H); Anal. Calcd. for $\mathrm{C}_{25} \mathrm{H}_{25} \mathrm{NO}$ (355); C, 84.50; H, 7.04; N, 3.94. Found; C, 84.57; $\mathrm{H}, 7.20 ; \mathrm{N}, 3.75 \%$.

2-Methyl-1-(7-methyl-2-phenyl-1H-indol-1-yl)propan-2-ol (12a). White needles; mp $68{ }^{\circ} \mathrm{C}$ (1:3 benzene/PE 60-80 $\left.{ }^{\circ} \mathrm{C}\right)$. IR (KBr) $v_{\max } 3370$, 3090, 2965, 1590, 1480, 1460, 1450, 1345 , 1030, $745 \mathrm{~cm}^{-1} ;{ }^{1} \mathrm{H} \mathrm{NMR}\left(90 \mathrm{MHz}, \mathrm{CDCl}_{3}, \mathrm{ppm}\right), \delta 1.20\left(6 \mathrm{H}, \mathrm{s}, 2 \mathrm{CH}_{3}\right), 2.30\left(3 \mathrm{H}, \mathrm{s}, \mathrm{CH}_{3}\right), 2.60$ $\left(1 \mathrm{H}, \mathrm{s}, \mathrm{OH}\right.$ exchangeable with $\left.\mathrm{D}_{2} \mathrm{O}\right), 4.0\left(2 \mathrm{H}, \mathrm{t}, J 7.5 \mathrm{~Hz}, \mathrm{CH}_{2}\right), 6.50(1 \mathrm{H}, \mathrm{s}, \mathrm{CH})$ and 6.80-8.10 (8H, m, Ar-H); Anal. Calcd. for $\mathrm{C}_{19} \mathrm{H}_{21} \mathrm{NO}$ (279); C, 81.72; H, 7.52; N, 5.01; Found; C, 81.53; $\mathrm{H}, 7.33 ; \mathrm{N}, 5.17 \%$.

2-Methyl-1-(7-methyl-2-phenyl-1H-indol-1-yl)butan-2-ol (12b). Reddish viscous oil; $n_{D}^{25}$ 1.568. IR (film) $v_{\max } 3450,3085,2970,1600,1480,1460,1450,1345,1020,740 \mathrm{~cm}^{-1} ;{ }^{1} \mathrm{H}$ NMR (90 MHz, $\left.\mathrm{CDCl}_{3}, \mathrm{ppm}\right), \delta 0.90\left(3 \mathrm{H}, \mathrm{t}, J 9.0 \mathrm{~Hz}, \mathrm{CH}_{3}\right), 1.30\left(3 \mathrm{H}, \mathrm{s}, \mathrm{CH}_{3}\right), 1.40(2 \mathrm{H}, \mathrm{t}, J$ $\left.9.0 \mathrm{~Hz}, \mathrm{CH}_{2}\right), 1.70\left(1 \mathrm{H}, \mathrm{s}, \mathrm{OH}\right.$ exchangeable with $\left.\mathrm{D}_{2} \mathrm{O}\right), 2.30\left(3 \mathrm{H}, \mathrm{s}, \mathrm{CH}_{3}\right), 3.80-4.10(2 \mathrm{H}, \mathrm{m}, J$ $\left.6.0 \mathrm{~Hz}, \mathrm{CH}_{2}\right), 6.60(1 \mathrm{H}, \mathrm{s}, \mathrm{CH})$ and 6.90-8.0 (8H, m, Ar-H); Anal. Calcd. for $\mathrm{C}_{20} \mathrm{H}_{23} \mathrm{NO}$ (293); C, 81.91; H, 7.84; N, 4.77; Found; C, 81.75; H, 8.24; N, 4.79\%.

1-(7-Methyl-2-phenyl-1H-indol-1-yl)-2-phenylpropan-2-ol (12c). Pale yellow viscous oil; $n_{D}^{25}$ 1.584. IR (film) $v_{\max } 3450,3085,2970,1600,1480,1460,1450,1345,1020,740 \mathrm{~cm}^{-1} ;{ }^{1} \mathrm{H}$ NMR $\left(90 \mathrm{MHz}, \mathrm{CDCl}_{3}, \mathrm{ppm}\right), \delta 1.60\left(3 \mathrm{H}, \mathrm{s}, \mathrm{CH}_{3}\right), 2.30\left(3 \mathrm{H}, \mathrm{s}, \mathrm{CH}_{3}\right), 1.40(1 \mathrm{H}, \mathrm{s}, \mathrm{OH}$ exchangeable with $\left.\mathrm{D}_{2} \mathrm{O}\right), 4.20-4.40\left(2 \mathrm{H}, \mathrm{m}, J 6.0 \mathrm{~Hz}, \mathrm{CH}_{2}\right), 6.50(1 \mathrm{H}, \mathrm{s}, \mathrm{CH})$ and 6.80-8.0 $(13 \mathrm{H}$, m, Ar-H); Anal. Calcd. for $\mathrm{C}_{24} \mathrm{H}_{23} \mathrm{NO}$ (341); C, 84.45; H, 6.74; N, 4.10; Found; C, 84.38; H, $6.79 ; \mathrm{N}, 4.37 \%$. 


\section{Cyclialkylation procedures}

The procedures described earlier for cyclialkylation of arylalkanols with $\mathrm{AlCl}_{3} / \mathrm{CH}_{3} \mathrm{NO}_{2}{ }^{30}$ and $\mathrm{PPA}^{31}$ were essentially followed. Purification of the crude isolated products with flash column chromatography (basic alumina, EtOAc/n-hexane, 1/1) gave the pure product. The conditions and yields for the products 1a-f and 2a-c are shown in Tables 2 and 3 while the physical constants and spectral data of the products are given below.

7,8-Dihydro-4,8,8-trimethyl-6H-indolo[2,1-a][2]benzazepine (1a). Reddish viscous oil; $n_{D}^{25} \quad 1.566 ; R_{f} 0.35$ (1:3 EtOAc/hexane). IR (film) $v_{\max } 3075,2985,1600,1565,1490,1445$, 1430, 1330, $1030 \mathrm{~cm}^{-1} ;{ }^{1} \mathrm{H}$ NMR (400 MHz, $\left.\mathrm{CDCl}_{3}, \mathrm{ppm}\right), \delta 1.30\left(6 \mathrm{H}, \mathrm{s}, 2 \mathrm{CH}_{3}\right), 2.0(2 \mathrm{H}, \mathrm{t}, J$ $\left.7.5 \mathrm{~Hz}, \mathrm{CH}_{2}\right), 2.30\left(3 \mathrm{H}, \mathrm{s}, \mathrm{CH}_{3}\right), 3.70\left(2 \mathrm{H}, \mathrm{t}, J 7.5 \mathrm{~Hz}, \mathrm{CH}_{2}\right), 6.50(1 \mathrm{H}, \mathrm{s}, \mathrm{CH})$ and 6.80-7.92 (7H, m, Ar-H); MS (EI, $70 \mathrm{eV}) \mathrm{m} / z(\%), 276\left(\mathrm{M}^{+}+1,44.8\right), 275\left(\mathrm{M}^{+}, 9.5\right), 274\left(\mathrm{M}^{+}-\mathrm{H}, 82.5\right)$, $260\left(\mathrm{M}^{+}-\mathrm{CH}_{3}, 100\right), 245$ (51.2), $230\left(\mathrm{M}^{+}-3 \mathrm{CH}_{3}, 21.5\right), 206$ (15.3), 192 (8.3), 178 (5.6), 166 (10.3), 151 (2.4), 109 (3.7), 91 (1.8), 77 (5.5); Anal. Calcd. for $\mathrm{C}_{20} \mathrm{H}_{21} \mathrm{~N}$ (275): C, 87.27; H, 7.63; N, 5.09. Found: C, 87.37; H, 7.85; N, 4.68\%.

8,8-Diethyl-7,8-dihydro-4-methyl-6H-indolo[2,1-a][2]benzazepine (1b). Pale yellow plates mp $90{ }^{\circ} \mathrm{C}$ (benzene); $R_{f} 0.42$ (1:3 EtOAc/hexane). IR (KBr) $v_{\max } 3075,2980,1610,1570,1490$, 1445, 1430, 1330, $1025 \mathrm{~cm}^{-1} ;{ }^{1} \mathrm{H}$ NMR (400 MHz, $\left.\mathrm{CDCl}_{3}, \mathrm{ppm}\right), \delta 0.90\left(6 \mathrm{H}, \mathrm{t}, J 9.0 \mathrm{~Hz}, 2 \mathrm{CH}_{3}\right)$, $1.50\left(2 \mathrm{H}, \mathrm{m}, J 9.0 \mathrm{~Hz}, 2 \mathrm{CH}_{2}\right), 2.0\left(2 \mathrm{H}, \mathrm{t}, J 7.5 \mathrm{~Hz}, \mathrm{CH}_{2}\right), 2.30\left(3 \mathrm{H}, \mathrm{s}, \mathrm{CH}_{3}\right), 3.70(2 \mathrm{H}, \mathrm{m}, J 7.5$ $\left.\mathrm{Hz}, \mathrm{CH}_{2}\right), 6.60(1 \mathrm{H}, \mathrm{s}, \mathrm{CH})$ and 6.90-8.0 (7H, m, Ar-H); MS (EI, $\left.70 \mathrm{eV}\right) \mathrm{m} / \mathrm{z}(\%), 203\left(\mathrm{M}^{+}, 7.5\right)$, $302\left(\mathrm{M}^{+}-\mathrm{H}, 100\right), 288\left(\mathrm{M}^{+}-\mathrm{CH}_{3}, 18.2\right), 274$ (40.5), $272\left(\mathrm{M}^{+}-2 \mathrm{CH}_{3}-\mathrm{H}, 45.2\right), 245\left(\mathrm{M}^{+}-2 \mathrm{C}_{2} \mathrm{H}_{5}\right.$, 11.8), $230\left(\mathrm{M}^{+}-2 \mathrm{C}_{2} \mathrm{H}_{5}-\mathrm{CH}_{3}, 17.4\right), 206$ (11.4), 192 (7.8), 179 (4.1), 166 (9.4), 151 (1.5), 109 (6.2), 91 (2.4), 77 (5.8); Anal. Calcd. for $\mathrm{C}_{22} \mathrm{H}_{25} \mathrm{~N}$ (303): C, 87.12; H, 8.25; N, 4.62. Found: C, $87.25 ; \mathrm{H}, 8.09 ; \mathrm{N}, 4.76 \%$.

7,8-Dihydro-4-methyl-8,8-diphenyl-6H-indolo[2,1-a][2]benzazepine (1c). White plates, mp $138{ }^{\circ} \mathrm{C}$ (benzene); $R_{f} 0.2$ (1:3 EtOAc/hexane). IR (KBr) $v_{\max } 3090,2976,1605,1590,1480$, 1450, 1425, 1330, $1030 \mathrm{~cm}^{-1} ;{ }^{1} \mathrm{H} \mathrm{NMR}\left(400 \mathrm{MHz}, \mathrm{CDCl}_{3}, \mathrm{ppm}\right), \delta 2.30\left(3 \mathrm{H}, \mathrm{s}, \mathrm{CH}_{3}\right), 2.70(2 \mathrm{H}$, t, $\left.J 7.5 \mathrm{~Hz}, \mathrm{CH}_{2}\right), 3.90\left(2 \mathrm{H}, \mathrm{t}, J 7.5 \mathrm{~Hz}, \mathrm{CH}_{2}\right), 6.60(1 \mathrm{H}, \mathrm{s}, \mathrm{CH})$ and 6.90-8.20 (17H, m, Ar-H); MS (EI, $70 \mathrm{eV}) \mathrm{m} / z(\%), 399\left(\mathrm{M}^{+}, 4.8\right), 398\left(\mathrm{M}^{+}-\mathrm{H}, 85.5\right), 384\left(\mathrm{M}^{+}-\mathrm{CH}_{3}, 32.4\right), 383$ (18.6), 322 ( $\left.\mathrm{M}^{+}-\mathrm{Ph}, 51.4\right), 245$ (20.6), $230\left(\mathrm{M}^{+}-2 \mathrm{Ph}-\mathrm{CH}_{3}, 22.7\right), 206$ (8.3), 192 (6), 178 (2.1), 166 (6.2), 151 (2.3), 109 (9.4), 91 (5.4), 77 (7.4); Anal. Calcd. for $\mathrm{C}_{30} \mathrm{H}_{25} \mathrm{~N}$ (399): C, 90.22; H, 6.26; N, 3.50 Found: C, 90.25; H, 6.36; N, 3.43\%.

8-Ethyl-7,8-dihydro-4,8-dimethyl-6H-indolo[2,1-a][2]benzazepine (1d). Brown viscous oil; $n_{D}^{25} \quad 1.574 ; R_{f} 0.52$ (1:3 EtOAc/hexane). IR (Film) $v_{\max } 3090,2970,1580,1490,1450,1420$, 1335, $1040 \mathrm{~cm}^{-1}$; ${ }^{1} \mathrm{H}$ NMR (400 MHz, $\left.\mathrm{CDCl}_{3}, \mathrm{ppm}\right), \delta 0.90\left(3 \mathrm{H}, \mathrm{t}, J 9.0 \mathrm{~Hz}, \mathrm{CH}_{3}\right), 1.30(3 \mathrm{H}, \mathrm{s}$, $\left.\mathrm{CH}_{3}\right), 1.60\left(2 \mathrm{H}, \mathrm{q}, J\right.$ 9.0 Hz, $\left.\mathrm{CH}_{2}\right), 1.80-2.20\left(2 \mathrm{H}, \mathrm{m}, J\right.$ 7.5 Hz, $\left.\mathrm{CH}_{2}\right), 2.30(1 \mathrm{H}, \mathrm{s}, \mathrm{CH}), 3.80$ $(2 \mathrm{H}, \mathrm{t}, J$ 7.5 Hz, CH$), 6.60(1 \mathrm{H}, \mathrm{s}, \mathrm{CH})$ and 6.90-7.90 (7H, m, Ar-H); MS (EI, $70 \mathrm{eV}) \mathrm{m} / \mathrm{z}(\%)$, $289\left(\mathrm{M}^{+}, 15.8\right), 274\left(\mathrm{M}^{+}-\mathrm{CH}_{3}, 72.4\right), 259\left(\left(\mathrm{M}^{+}-\mathrm{C}_{2} \mathrm{H}_{5}-\mathrm{H}, 100\right), 245\left(\mathrm{M}^{+}-\mathrm{CH}_{3}-\mathrm{C}_{2} \mathrm{H}_{5}, 45.2\right), 244\right.$ (88.3), $230\left(\left(\mathrm{M}^{+}-2 \mathrm{CH}_{3}-\mathrm{C}_{2} \mathrm{H}_{5}, 14.6\right), 206\right.$ (62.4), 204 (14.2), 191 (14.2), 177 (4.3), 167 (18.2), 151 (7.3), 109 (5.8), 90 (4.5), 77 (5.7), 66 (4.2); Anal. Calcd. for $\mathrm{C}_{21} \mathrm{H}_{23} \mathrm{~N}$ (289): C, 87.19; H, 7.95; N, 4.84. Found: C, 87.40; H, 7.62; N, $4.92 \%$. 
8-Ethyl-7,8-dihydro-4-methyl-8-phenyl-6H-indolo[2,1-a][2]benzazepine (1e). White crystals, $\mathrm{mp} 72{ }^{\circ} \mathrm{C}$ (benzene); $R_{f} 0.43$ (1:3 EtOAc/hexane). IR (KBr) $v_{\max } 3075,2965,1580,1500,1430$, 1415, 1330, $1020 \mathrm{~cm}^{-1}$; ${ }^{1} \mathrm{H}$ NMR (400 MHz, $\left.\mathrm{CDCl}_{3}, \mathrm{ppm}\right), \delta 0.90\left(3 \mathrm{H}, \mathrm{t}, J 9.0 \mathrm{~Hz}, \mathrm{CH}_{3}\right), 1.90$ $\left(2 \mathrm{H}, \mathrm{t}, J 9.0 \mathrm{~Hz}, \mathrm{CH}_{2}\right), 2.20-2.50\left(2 \mathrm{H}, \mathrm{m}, J 7.5 \mathrm{~Hz}, \mathrm{CH}_{2}\right), 2.30\left(3 \mathrm{H}, \mathrm{s}, \mathrm{CH}_{3}\right), 3.80(2 \mathrm{H}, \mathrm{t}, J 7.5$ $\left.\mathrm{Hz}, \mathrm{CH}_{2}\right), 6.60(1 \mathrm{H}, \mathrm{s}, \mathrm{CH})$ and 7.10-8.20 (7H, m, Ar-H); MS (EI, $\left.70 \mathrm{eV}\right) \mathrm{m} / \mathrm{z}(\%), 352\left(\mathrm{M}^{+}+1\right.$, 27.4), $351\left(\mathrm{M}^{+}, 6.2\right), 335\left(\mathrm{M}^{+}-\mathrm{H}-\mathrm{CH}_{3}, 62.8\right), 322\left(\mathrm{M}^{+}-\mathrm{C}_{2} \mathrm{H}_{5}, 100\right), 307$ (34.6), $274\left(\mathrm{M}^{+}-\mathrm{Ph}\right.$, 18.5), 245 (33.8), 230 (42.6), 206 (7.4), 191 (14.2), 177 (12.6), 167 (8.4), 151 (3.7), 109 (7.4), 90 (17.4), 77 (5.3); Anal. Calcd. for $\mathrm{C}_{26} \mathrm{H}_{25} \mathrm{~N}$ (351): C, 88.88; H, 7.12; N, 3.98. Found: C, 88.63; H, $7.28 ; \mathrm{N}, 4.15 \%$.

7,8-Dihydro-4,8-dimethyl-8-phenyl-5H-indolo[2,1-a][2]benzazepine (1f). Brownish viscous oil; $n_{D}^{25} 1.542 ; R_{f} 0.47$ (1:3 EtOAc/hexane); IR (film) $v_{\max } 3085,2984,1592,1505,1470,1450$, 1430, 1330, $1020 \mathrm{~cm}^{-1} ;{ }^{1} \mathrm{H}$ NMR (400 MHz, $\left.\mathrm{CDCl}_{3}, \mathrm{ppm}\right), \delta 1.70\left(3 \mathrm{H}, \mathrm{s}, \mathrm{CH}_{3}\right), 2.20-2.50(2 \mathrm{H}$, m, J 7.5 Hz, $\left.\mathrm{CH}_{2}\right), 2.30\left(3 \mathrm{H}, \mathrm{s}, \mathrm{CH}_{3}\right), 3.80\left(2 \mathrm{H}, \mathrm{t}, J 7.5 \mathrm{~Hz}, \mathrm{CH}_{2}\right), 6.60(1 \mathrm{H}, \mathrm{s}, \mathrm{CH})$ and 6.908.10 (12H, m, Ar-H); MS (EI, $70 \mathrm{eV}) \mathrm{m} / z(\%), 339\left(\mathrm{M}^{+}+2,48.6\right), 337\left(\mathrm{M}^{+}, 7.4\right), 336\left(\mathrm{M}^{+}-\mathrm{H}\right.$, 100), $322\left(\mathrm{M}^{+}-\mathrm{CH}_{3}, 77.4\right), 307\left(\mathrm{M}^{+}-2 \mathrm{CH}_{3}, 27.3\right), 260\left(\mathrm{M}^{+}-\mathrm{Ph}, 41.7\right), 245\left(\mathrm{M}^{+}-\mathrm{Ph}-\mathrm{CH}_{3}, 28.5\right)$, 230 (27.2), 206 (11.4), 190 (24.7), 177 (9.6), 166 (7.4), 151 (4.4), 109 (6.4), 90 (12.5), 77 (3.7); Anal. Calcd. for $\mathrm{C}_{25} \mathrm{H}_{23} \mathrm{~N}$ (337): C, 89.02; H, 6.82; N, 4.15. Found: C, 89.15; H, 6.92; N, 3.94\%.

6,7-Dihydro-4,7,7-trimethylindolo[2,1-a]isoquinoline (2a). White crystals, mp $140{ }^{\circ} \mathrm{C}$ (benzene); $R_{f} 0.36$ (1:3 EtOAc/hexane). IR (KBr) $v_{\max } 3080,2975,1610,1550,1480,1435$, 1335, $1025 \mathrm{~cm}^{-1} ;{ }^{1} \mathrm{H}$ NMR (400 MHz, $\left.\mathrm{CDCl}_{3}, \mathrm{ppm}\right), \delta 1.70\left(6 \mathrm{H}, \mathrm{s}, 2 \mathrm{CH}_{3}\right), 2.30\left(3 \mathrm{H}, \mathrm{s}, \mathrm{CH}_{3}\right)$, $4.10\left(2 \mathrm{H}, \mathrm{s}, \mathrm{CH}_{2}\right), 6.60(1 \mathrm{H}, \mathrm{s}, \mathrm{CH})$ and 6.80-8.0 (7H, m, Ar-H); MS (EI, $\left.70 \mathrm{eV}\right) \mathrm{m} / \mathrm{z}(\%), 262$ $\left(\mathrm{M}^{+}+1,57.2\right), 261\left(\mathrm{M}^{+}, 6.8\right), 246\left(\mathrm{M}^{+}-\mathrm{CH}_{3}, 100\right), 231$ (24.7), $216\left(\mathrm{M}^{+}-3 \mathrm{CH}_{3}, 82.4\right), 204$ (16.7), 192 (25.3), 178 (20.4), 166 (13.5), 151 (4.6), 109 (4.7), 91 (17.4), 77 (3.4), 66 (2.7); Anal. Calcd. for $\mathrm{C}_{19} \mathrm{H}_{19} \mathrm{~N}$ (261): C, 87.35; H, 7.27; N, 5.36. Found: C, 87.17; H, 7.42; N, 5.25\%.

7-Ethyl-6,7-dihydro-4,7-dimethylindolo[2,1-a]isoquinoline (2b). Pale yellow crystals, mp 122 ${ }^{\circ} \mathrm{C}$ (benzene); $R_{f} 0.38$ (1:3 EtOAc/hexane). IR (KBr) $v_{\max } 3095,2979,1606,1580,1480,1440$, $1335,1020 \mathrm{~cm}^{-1} ;{ }^{1} \mathrm{H} \mathrm{NMR}\left(400 \mathrm{MHz}, \mathrm{CDCl}_{3}, \mathrm{ppm}\right), \delta 0.90\left(6 \mathrm{H}, \mathrm{t}, J 9.0 \mathrm{~Hz}, \mathrm{CH}_{3}\right), 1.30(4 \mathrm{H}, \mathrm{m}$, $\left.J 9.0 \mathrm{~Hz}, \mathrm{CH}_{2}\right), 1.60\left(2 \mathrm{H}, \mathrm{q}, J 9.0 \mathrm{~Hz}, \mathrm{CH}_{2}\right), 2.30\left(3 \mathrm{H}, \mathrm{s}, \mathrm{CH}_{3}\right), 3.80-4.10(2 \mathrm{H}, \mathrm{m}, J \mathrm{~Hz}$, $\left.\mathrm{CH}_{2}\right), 6.60(1 \mathrm{H}, \mathrm{s}, \mathrm{CH})$ and 6.80-8.0 (7H, m, Ar-H); MS (EI, $\left.70 \mathrm{eV}\right) \mathrm{m} / \mathrm{z}(\%), 276\left(\mathrm{M}^{+}+1,30.6\right)$, $275\left(\mathrm{M}^{+}, 4.7\right), 260\left(\mathrm{M}^{+}-\mathrm{CH}_{3}, 92.5\right), 246\left(\mathrm{M}^{+}-\mathrm{C}_{2} \mathrm{H}_{5}, 25.3\right), 245\left(\mathrm{M}^{+}-\mathrm{C}_{2} \mathrm{H}_{5}-\mathrm{H}, 100\right), 231$ $\left(\mathrm{M}^{+}-\mathrm{CH}_{3}-\mathrm{C}_{2} \mathrm{H}_{5}, 53.7\right), 216$ (20.4), 202 (26), 188 (13.7), 177 (5.3), 166 (12.5), 151 (2.4), 109 (5.4), 90 (4.7), 77 (4.2), 66 (3.1); Anal. Calcd. for $\mathrm{C}_{20} \mathrm{H}_{21} \mathrm{~N}$ (275): C, 87.27; H, 7.63; N, 5.09. Found: C, 87.45; H, 7.53; N, 4.83\%.

6,7-Dihydro-4,7-dimethyl-7-phenylindolo[2,1-a]isoquinoline (2c). White needles, mp $107{ }^{\circ} \mathrm{C}$ (benzene); $R_{f} 0.25$ (1:3 EtOAc/hexane). IR (KBr) $v_{\max } 3090,2980,1610,1575,1490,1450$, 1342, $1033 \mathrm{~cm}^{-1} ;{ }^{1} \mathrm{H}$ NMR (400 MHz, $\left.\mathrm{CDCl}_{3}, \mathrm{ppm}\right), \delta 1.60\left(3 \mathrm{H}, \mathrm{s}, \mathrm{CH}_{3}\right), 2.30\left(3 \mathrm{H}, \mathrm{s}, \mathrm{CH}_{3}\right)$, 4.10-4.40 (2H, m, J 7.5 Hz, CH $), 6.60(1 \mathrm{H}, \mathrm{s}, \mathrm{CH})$ and 6.80-8.20 (12H, m, Ar-H); MS (EI, 70 eV) $m / z(\%), 324\left(\mathrm{M}^{+}+1,30.4\right), 323\left(\mathrm{M}^{+}, 5.2\right), 322\left(\mathrm{M}^{+}-\mathrm{H}, 100\right), 308\left(\mathrm{M}^{+}-\mathrm{CH}_{3}, 62.8\right), 293$ $\left(\mathrm{M}^{+}-2 \mathrm{CH}_{3}, 37.5\right), 246\left(\mathrm{M}^{+}-\mathrm{Ph}, 69.3\right), 245\left(\mathrm{M}^{+}-\mathrm{Ph}-\mathrm{H}, 40.2\right), 231$ (15.4), 216 (14.7), 202 (10), 
190 (23.5), 177 (8.6), 166 (11.4), 151 (5.3), 109 (5.2), 91 (16.7), 77 (2.5); Anal. Calcd. for $\mathrm{C}_{24} \mathrm{H}_{21} \mathrm{~N}$ (323): C, 89.16; H, 6.50; N, 4.33. Found: C, 89.50; H, 6.32; N, 4.15\%.

\section{Acknowledgements}

The authors are grateful for the support and facilities offered by the Chemistry Department, Faculty of Science, Assiut University, Assiut, Egypt.

\section{References}

1. (a) Thevissen, K.; Marchand, A.; Chaltin, P.; Meert, E. M. K.; Cammue, B. P. A. Curr. Med. Chem. 2009, 16, 2205. (b) Conchon, E.; Anizon, F.; Aboab, B.; Prudhomme, M. J. Med. Chem. 2007, 50, 4669. (c) Bressy, C.; Alberico, D.; Lautens, M. J. Am. Chem. Soc. 2005, $127,13148$.

2. (a) Humphrey, G. R.; Kuethe, J. T. Chem. Rev. 2006, 106, 2875. (b) Sharma, V.; Lansdell, T. A.; Jin, G.; Tepe, J. J. J. Med. Chem. 2004, 47, 3700.

3. (a) Gil-Tumes, M. S.; Hay, M. E.; Fenical, W. Science 1989, 246, 116. (b) Rustagi, V.; Aggarwal, T. I.; Verma, A. K.; Green Chem. 2011, 13, 1640. (c) Djura, P.; Faulkner, D. J. J. Org. Chem. 1980, 45, 735. (d) Padwa, A.; Bur, S. K.; Danca, D. M.; Ginn, J. D.; Lynch, S. M. Synlett 2002, 851.

4. (a) Chacun-Lefèvre, L.; Joseph, B.; Mérour, J. Y. Synlett 2001, 848. (b) Meanwell, N. A.; Gentles, R. G.; Ding, M.; Bender, J. A.; Kadow, J. F.; Hewawasam, P.; Hudyma, T. W.; Zheng, X. U.S. Patent 2,007,184,024, 2007; Chem. Abstr. 2007, 147, 257667.

5. Faust, R.; Garratt, P. J.; Jones, R.; Yeh, L. K. J. Med. Chem. 2000, 43, 1050.

6. Verma, A. K.; Kesharwani, T.; Singh, J.; Tandon, V.; Larock, R. C. Angew. Chem. Int. Ed. 2009, 48, 1138.

7. Sánchez-Sancho, F.; Mann, E.; Herradón, B. Synlett 2000, 509.

8. Lötter, A. N. C.; Pathak, R.; Sello, T. S.; Fernandes, M. A.; van Otterlo, W. A. L.; de Koning, C. B. Tetrahedron 2007, 63, 2263 and references therein.

9. Ewing, J.; Hughes, G. K.; Ritchie, E.; Taylor, W. C. Nature 1952, 169, 618.

10. (a) Goldbrunner, M.; Loid, G.; Polossek, T.; Mannschreck, A.; von Angerer, E. J. Med. Chem. 1997, 40, 3524. (b) Ambros, R.; von Angerer, S.; Wiegrebe, W. Arch. Pharm. 1988, $321,743$.

11. (a) Boden, N.; Bissell, R.; Clements, J.; Movaghar, B. Liq. Cryst. Today 1996, 6, 1. (b) Ahmed, E.; Briseno, A. L.; Xia, Y.; Jenekhe, S. A. J. Am. Chem. Soc. 2008, 130, 1118.

12. Kozikowski, A. P.; Ma, D. Tetrahedron Lett. 1991, 32, 3317.

13. Kozikowski, A. P.; Ma, D.; Brewer, J.; Sun, S.; Costa, E.; Romeo, E.; Guidotti, H. J. Med. Chem. 1993, 36, 2908. 
14. Lee, H. S.; Kim, S. H.; Kim, T. H., Kim, J. N. Tetrahedron Lett. 2008, 49, 1773.

15. Lee, H. S.; Kim, K. H.; Kim, Y. M.; Kim, J. N. Bull. Korean Chem. Soc. 2010, 31, 1761.

16. For the Pd-mediated reactions involving Baylis-Hillman adducts, see: (a) Gowrisankar, S.; Lee, H. S.; Lee, K. Y.; Lee, J.-E.; Kim, J. N. Tetrahedron Lett. 2007, 48, 8619 and further references cited therein. (b) Basavaiah, D.; Rao, A. J.; Satyanarayana, T. Chem. Rev. 2003, 103, 811. (c) Declerck, V.; Ribiere, P.; Nedellec, Y.; Allouchi, H.; Martinez, J.; Lamaty, F. Eur. J. Org. Chem. 2007, 201. (d) Ribiere, P.; Declerck, V.; Nedellec, Y.; Yadav-Bhatnagar, N.; Martinez, J.; Lamaty, F. Tetrahedron 2006, 62, 10456. (e) Vasudevan, A.; Tseng, P. -S.; Djuric, S. W. Tetrahedron Lett. 2006, 47, 8591.

17. (a) Orito K.; Harada, R.; Uchiito, S.; Tokuda, M. Org. Lett. 2000, 2, 1799. (b) Orito, K.; Miyazawa, M.; Kanbayashi, R.; Tokuda, M.; Suginome, H. J. Org. Chem. 1999, 64, 6583.

18. Sharma, S. K.; Sharma, S.; Agarwal, P. K.; Kundu, B. Eur. J. Org. Chem. 2009, 1309 and references therein.

19. (a) Kundu, B.; Sawant, D.; Chhabra, R. J. Comb. Chem. 2005, 7, 317. (b) Duggineni, S.; Sawant, D.; Saha, B.; Kundu, B., Tetrahedron 2006, 62, 3228. (c) S. Sharma, B. Saha, D. Sawant, B. Kundu, J. Comb. Chem. 2007, 9, 783-792.

20. Meyers, A. I.; Sielecki, T. M. J. Am. Chem. Soc. 1991, 113, 2789.

21. (a) Harley-Mason, J. J. Chem. Soc. 1953, 1465. (b) Benington, F.; Morin, R. D. J. Org. Chem. 1967, 32, 1050.

22. Ninomiya, I.; Yasui, J.; Kiguchi, T. Heterocycles 1977, 6, 1855.

23. Takano, S.; Satoh, S.; Ogasawara, K. Heterocycles 1987, 26, 1483.

24. (a) Yasuda, S.; Hirasawa, T.; Yoshida, H.; Hanaoka, M. Chem. Pharm. Bull. 1989, 37, 1682. (b) Orito, K.; Uchiito, S.; Satoh, Y.; Tatsuzawa, T.; Harada, R.; Tozuda, M. Org. Lett. 2000, 2, 307. (c) Bennasar, M.-L.; Roca, T.; Ferrando, F. Org. Lett. 2004, 6, 759.

25. (a) Harada, R.; Nishida, N.; Uchiito, S.; Onozaki, Y.; Kurono, N.; Senboku, H.; Masao, T.; Ohkuma, T.; Orito, K. Eur. J. Org. Chem. 2012, 366. (b) Gilchrist, T. L.; Kemmitt, P. D. Tetrahedron 1997, 53, 4447.

26. (a) Wasilke, J. -C.; Obrey, S. J.; Baker, T.; Bazan, G. C. Chem. Rev. 2005, 105, 1001. (b) Nicolaou, K. C.; Chen, J. S. Chem. Soc. Rev. 2009, 38, 2993 (c) Kirsch, S. F. Synthesis 2008, 3183.

27. Kirsch, G. H. Curr. Org. Chem. 2001, 5, 507.

28. Knölker, H. -J.; Reddy, K. R. Chem. Rev. 2002, 102, 4303.

29. (a) Olah, G. A. In Friedel-Crafts Chemistry; Olah, G. A., Ed.; Wiley: NewYork, NY, 1973.

(b) Barclay, L. R. C. In Friedel-Crafts and Related Reactions; Olah, G. A. Ed.; Interscience, New York, 1964, Vol. II, Chap. 22 and references therein. (c) Roberts, R. M.; Khalaf, A. A. Friedel-Crafts Chemistry: A Century of Discovery; Marcel Dekker: New York, NY, 1984. (d) Bandini, M.; Melloni, A.; Tommasi, S.; Umani-Ronchi, A. Synlett 2005, 1199. (e) Poulsen, T. B.; Jørgensen, K. A. Chem. Rev. 2008, 108, 2903. (f) Terrasson, V.; Marcia de Figueiredo, R.; Campagne, J. M. Eur. J. Org. Chem. 2010, 14, 2635.

30. Khalaf, A. A.; Roberts, R. M. J. Org. Chem. 1972, 37, 4227 and references therein. 
31. Khalaf, A. A.; Makki, M. S. I. T.; Kabli, R. A. J. Indian Chem. Soc. 1997, 74, 148.

32. Khalaf, A. A.; Awad, I. M.; El-Emary, T. I.; Abd El-Aal, H. A. K., J. Indian Chem. Soc. 2008, 85, 6.

33. Khalaf, A. A.; Awad, I. M.; El-Emary, T. I.; Abd El-Aal, H. A. K. J. Indian Chem. Soc. 2006, 83, 10.

34. Khalaf, A. A.; El-Khawaga, A. M.; Awad, I. M.; Abd El-Aal, H. A. K. Arkivoc 2009, (xiv), 314.

35. Khalaf, A. A.; Awad, I. M.; El-Emary, T. I.; Abd El-Aal, H. A. K. J. Indian Chem. Soc. 2010, 87, 595.

36. Khalaf, A. A.; El-Khawaga, A. M.; Awad, I. M.; Abd El-Aal, H. A. K. Arkivoc 2010, (x), 338.

37. Kamlet, M. J.; Dacons, J. C. J. Org. Chem., 1961, 26, 220.

38. Fieser, L. F.; Seligman, A. M. J. Am. Chem. Soc. 1936, 58, 2483.

39. Cason, J.; Prout, F. S. J. Am. Chem. Soc. 1944, 66, 46.

40. La Forge, F. B. J. Am. Chem. Soc. 1928, 50, 2484.

41. Blume, L. S. and Lindwall, A. J. Org. Chem. 1945, 10, 255. 